Análisis de la validez convergente de la Versión reducida en español del cuestionario de actividad fisica en el tiempo libre de Minnesota (VREM) y de la versión española del Cuestionario internacional de actividad fisica en personas mayores (IPAQ-E)

\title{
Analysis of the convergent validity of the Spanish short version of the Minnesota Leisure Time Physical Activity Questionnaire (VREM) and the Spanish version of the International Physical Activity Questionnaire in elderly people (IPAQ-E)
}

https://doi.org/10.23938/ASSN.0633

D. Martínez-Aldao' ${ }^{1}$ J.C. Diz ${ }^{2}$, S. Varela ${ }^{3}$, C. Ayán ${ }^{2}$

\begin{abstract}
RESUMEN
Fundamento. Los cuestionarios Versión reducida en español del cuestionario de actividad física en el tiempo libre de Minnesota (VREM) y Cuestionario internacional de actividad física adaptado a las personas mayores (IPAQ-E) se consideran herramientas de utilidad cuando se pretende cuantificar la actividad física (AF) que la población geriátrica realiza en nuestro país. Sin embargo, son escasos los estudios realizados al respecto de sus propiedades psicométricas. Por ello, esta investigación pretende aportar información sobre la validez convergente de ambos cuestionarios, analizando el grado de asociación que muestran con la condición física (CF).
\end{abstract}

Material y Métodos. Un total de 105 personas (edad media: 76,77 (DE=6,01) años; 84,8\% mujeres), contestaron ambos cuestionarios y realizaron la batería Senior Fitness Test (SFT) al objeto de recoger información sobre su nivel de CF.

Resultados. Se encontraron correlaciones significativas entre la SFT y los ítems del VREM relativos al gasto energético derivado de caminar $(\mathrm{r}=0,227)$ o de realizar actividades deportivas o baile $(r=0,235)$, y el gasto energético total estimado $(r=0,314)$. El IPAQ-E no estableció asociaciones significativas con ninguna de las variables analizadas. Aunque a priori se observó una cierta correlación entre el VREM y el IPAQ-E $(\mathrm{r}=0,447, \mathrm{p}<0,001)$, y la diferencia media entre ambos parámetros fue nula $(\mathrm{p}=0,553)$, se consideró que los cuestionarios mostraron un grado de concordancia escaso, debido a la gran amplitud encontrada en el rango de diferencias

Conclusiones. Se aconseja el empleo del cuestionario VREM para estimar la cantidad de AF en población geriátrica, ya que presenta cierto grado de asociación, si bien débil, con el nivel de CF.

Palabras clave. Cuestionarios. Psicometría. Tercera edad. Condición física. Actividad física.

\begin{abstract}
Background. In our country the Spanish short version of the Minnesota Leisure Time Physical Activity Questionnaire (VREM) and the International Physical Activity Questionnaire in elderly people (IPAQ-E) are considered useful tools for estimating the amount of physical activity (PA) performed by the geriatric population. However, few studies have been carried out on their psychometric properties. Therefore, this research aims to provide information on their convergent validity by analysing the degree of association that both questionnaires show with physical fitness level.
\end{abstract}

Material and Methods. A total of 105 people (mean age: 76.77 ( $\mathrm{SD}=6.01$ ) years; $84.8 \%$ women), answered both questionnaires and performed the Senior Fitness Test (SFT), in order to assess their physical fitness level.

Results. Significant correlations were found between the SFT and the VREM items related to energy expenditure derived from walking $(r=0.227)$ or from performing sports activities or dancing $(r=0.235)$, and the estimated total energy expenditure $(\mathrm{r}=0.314)$. The IPAQ-E did not establish significant associations with any of the variables analysed. Although a certain a priori correlation was observed between the VREM and the IPAQ-E $(r=0.447, \mathrm{p}<0.001)$ and the mean difference between both parameters was nil $(\mathrm{p}=0.553)$, it was considered that the questionnaires showed a lack of agreement due to the great width found in the range of differences.

Conclusions. The use of the VREM questionnaire is recommended for estimating the amount of PA performed by the geriatric population, as it shows a certain degree of association, albeit weak, with the level of physical fitness.

Keywords. Questionnaire. Psychometry. Older Adults. Physical Condition. Physical Fitness.
1. Facultad de Ciencias de la Educación y el Deporte. Universidad de Vigo.

2. Well-Move Research Group. Facultad de Ciencias de la Educación y el Deporte. Universidad de Vigo.

3. Grupo de Investigación HealthyFit. Instituto de Investigación Sanitaria Galicia Sur. SERGAS-UVIGO. Pontevedra.

Recepción: 29/01/2019

Aceptación provisional: 26/03/2019

Aceptación definitiva: 09/04/2019

\section{Correspondencia:}

Daniel Martínez Aldao

Facultad de Ciencias de la Educación y el Deporte Universidad de Vigo

Campus A Xunqueira s/n

36004 Pontevedra

E-mail: damartinez@alumnos.uvigo.es 


\section{INTRODUCCIÓN}

El sedentarismo es considerado un factor de riesgo para la salud, por lo que cada vez es más importante que los profesionales de este campo aconsejen la práctica de actividad física (AF) a sus pacientes ${ }^{1}$. Esta estrategia es especialmente relevante para las personas de la tercera edad, en las que la realización de $\mathrm{AF}$ se ha relacionado con incrementos tanto en su capacidad funcional como en su calidad y en su esperanza de vida ${ }^{2-4}$. Por tanto, los profesionales de la salud necesitan disponer de herramientas que les permitan cuantificar la cantidad de AF que estas personas estiman realizar, con el fin de identificar a aquellos pacientes en los que la prescripción de la misma sea una acción prioritaria.

A este respecto, los cuestionarios están considerados como instrumentos de gran interés, tanto por su bajo coste económico y facilidad de administración, como por aportar información que permite cuantificar y clasificar el nivel de AF que el encuestado dice realizar ${ }^{5,6}$. De entre los cuestionarios que sirven a este propósito, que han sido validados en población nacional y cuyas propiedades psicométricas han sido previamente informadas, hay dos que merecen especial mención: Versión reducida en español del cuestionario de actividad física en el tiempo libre de Minnesota (VREM), que sobresale frente a otros cuestionarios similares por su grado de validez de constructo $^{7}$, y el Cuestionario internacional de actividad física (IPAQ), por ser mundialmente empleado, y del que existe una versión específicamente diseñada para ser administrado en personas mayores, el IPAQ-E ${ }^{8}$.

El VREM mostró una elevada validez de criterio $(k=0,93)$ y buena fiabilidad testretest $(k=0,88)$. En el caso del IPAQ-E, se observó una elevada fiabilidad interobservador $(\mathrm{CCI}=0,91)$ y una consistencia interna adecuada para cada una de sus dimensiones $(\alpha=0,81-0,88)$. Sin embargo, se ha considerado la necesidad de llevar a cabo un mayor número de investigaciones que permitan incrementar la evidencia científica existente al respecto ${ }^{9,10}$.

Una de los procedimientos metodológicos de gran utilidad para identificar la validez de un cuestionario diseñado para estimar la cantidad de AF realizada, es comparar su resultado con el nivel de condición física (CF) de los encuestados ${ }^{11-13}$. Este proceso, que sirve para determinar la validez convergente de este tipo de herramientas, se basa tanto en el hecho de que la $\mathrm{AF}$ y la CF son factores de riesgo directamente ligados con la salud, como en la gran relación que existe entre ellos ${ }^{14}$. Dadas las limitaciones que, por lo general, presentan los cuestionarios de estimación de la $\mathrm{AF}$, principalmente relacionadas con el sesgo de recuerdo y la capacidad del encuestado para entender ciertos conceptos relacionados con la misma, es de gran interés el profundizar en el estudio de sus propiedades psicométricas. Esto facilitará a los profesionales de la salud la elección del tipo de cuestionario de $\mathrm{AF}$ que mejor se adecúe a sus intereses.

De acuerdo a lo aquí expuesto, esta investigación pretende determinar el grado de asociación que presentan el VREM y el IPAQ-E con la CF y sus componentes, a fin de aportar información sobre la validez convergente de los mismos.

\section{MÉTODOS}

Se realizó un estudio transversal mediante un muestreo de conveniencia.

Los participantes del estudio fueron reclutados a través de un programa de gimnasia de mantenimiento ofertado por el ayuntamiento de Vigo. Los criterios de inclusión para tomar parte en la investigación fueron: tener más de 65 años de edad, no presentar problemas de memoria y tener autorización médica para tomar parte en programas de gerontogimnasia. Se excluyeron del estudio a todas aquellas personas que presentaron algún tipo de patología que impidiese la realización de las pruebas incluidas en el mismo y/o que expresaron el deseo, de manera personal o a través de sus familiares, de no participar en la investigación. Los participantes fueron informados sobre el objetivo y características del estudio y se obtuvo su consentimiento para tomar parte en el mismo. El diseño de la investigación 
fue aprobado por el Comité de Ética de la Facultad de Ciencias de la Educación y el Deporte (Universidad de Vigo).

La estimación preliminar del tamaño de muestra, asumiendo un $25 \%$ de pérdidas durante el seguimiento, un nivel de significación del $5 \%$ y una potencia del $80 \%$, fue de 100 sujetos para detectar, mediante una comparación de datos relacionados, una diferencia inferior a 1.000 MET (metabolic energy turnover, gasto energético en reposo), asumiendo una desviación estándar (DE) de 3.000 MET.

Se registraron la edad y el sexo de los participantes, y se determinó su índice de masa corporal (IMC), tras tallarlos con un tallímetro portátil y pesarlos con una báscula digital.

El cuestionario VREM demanda información sobre seis actividades realizadas en el último mes (o mes habitual) para conocer el nivel de AF realizada: caminar, trabajar en el huerto, hacer deporte o bailar, subir escaleras, ir a comprar a pie y limpiar la casa. De acuerdo con la cantidad de minutos destinados a cada actividad y a la frecuencia con que las mismas son realizadas a lo largo de un año, se estima el gasto energético durante 14 días, expresado en MET por minuto por 14 días (MET-min-14 días). El VREM permite distinguir entre personas muy activas (más de 5.000 MET-min-14 días), activas (entre 3.000 y 4.999), moderadamente activas (entre 1.250 y 2.999) y sedentarias (inferior a 1.250) ${ }^{9}$.

La versión en español del IPAQ-E consta de siete preguntas abiertas referidas a las actividades físicas realizadas por los adultos mayores en los últimos siete días, obteniendo el tiempo en reposo, el tiempo caminando y el tiempo realizando actividades moderadas o vigorosas. Una vez completado el cuestionario, y utilizando unos valores MET de referencia (caminar: 3,3 MET, AF moderada: 4 MET, AF vigorosa: 8 MET), se calcula el nivel de AF semanal multiplicando los MET por los minutos y días empleados en cada actividad. Sumados los valores se obtienen los MET por minuto por semana (MMS) y se distribuyen los sujetos en tres categorías de actividad ${ }^{10}$ : alta (3 o más días de $\mathrm{AF}$ vigorosa o que acumule 1.500 MMS o siete o más días de cualquier combinación que alcance los 7.000 MMS), media (3 o más días de $\mathrm{AF}$ vigorosa al menos $20 \mathrm{~min} /$ día, o 5 o más días de AF moderada, o caminar al menos $30 \mathrm{~min} /$ día, o cualquier combinación de AF que alcance 600 MMS), y baja (no se alcanzan los 600 MMS).

La valoración de la CF se realizó mediante la batería Senior Fitness Test ${ }^{15}$ (SFT), que incluye seis pruebas destinadas a valorar los siguientes componentes de la CF en personas mayores:

I. Fuerza del tren inferior (30-second chair stand): sentarse y levantarse de una silla; veces en 30 s.

II. Fuerza del tren superior (arm curl): flexiones completas del brazo realizadas sentado en una silla y con la espalda recta; veces en 30 s.

III. Flexibilidad de tren inferior (chair sit-and-reach): flexión del tronco sentado en el borde de la silla, con una pierna flexionada y la otra extendida, con los brazos extendidos y las manos juntas se intenta superar los dedos de los pies de la pierna extendida; centímetros que se sobrepasa el pie (positivos) o que no se alcanza (negativos).

IV. Capacidad cardiorrespiratoria (twominute step): número de pasos completos (izquierda y derecha) en dos minutos; se contabiliza el número de veces que la rodilla derecha alcanza el punto medio del muslo.

V. Flexibilidad de tren superior (backscratch): de pie y con la mano preferida sobre el mismo hombro, tratar de llevarla hacia el centro de la espalda tan lejos como sea posible mientras que el otro brazo se colocará en la espalda rodeando la cintura y, llevándola lo más lejos posible, intentar que los dedos medios de ambas manos se toquen; centím.etros que se solapan los dedos (positivos) o distancia entre ellos (negativos)

VI. Agilidad y equilibrio dinámico (8foot up-and-go): levantarse de una silla, caminar rodeando un cono situado a 2,44 metros y volver a sentarse; segundos en realizarlo. 
Las valoraciones tuvieron lugar entre los meses de mayo y junio del año 2018 y se realizaron en bloques de tres semanas, ante la imposibilidad de valorar a toda la muestra en una misma semana. Durante la primera semana los participantes cumplimentaron el cuestionario IPAQ-E individualmente y con la ayuda de un entrevistador que facilitó la comprensión de las preguntas formuladas. La semana siguiente se administró la batería SFT y durante la tercera semana los participantes cumplimentaron el VREM, mediante entrevista individualizada. Ambos cuestionarios fueron realizados por el mismo entrevistador, que además supervisó la valoración de la condición física de los participantes, contando para ello con la colaboración de dos personas más, todos ellos graduados en Ciencias de la Actividad Física y el Deporte.

Las variables cuantitativas se expresaron como media y DE, o como mediana y rango intercuartil (RIC, que incluye los valores del primer y tercer cuartil) si no tenían distribución normal comprobada con el test de Kolmogorov-Smirnov, y las cualitativas se expresaron en frecuencia y porcentaje. Para la comparación entre las distintas variables cuantitativas se utilizó el coeficiente de correlación de Pearson, siendo interpretado el grado de asociación entre las mismas del siguiente modo: $\geq 0,8$ excelente, 0,79-0,60 buena, 0,59-0,30 aceptable $\mathrm{y} \leq 0,29$ débil $^{16}$. Las pruebas t-Student y ANOVA se aplicaron para comparar dos o más grupos de variables con distribución normal, y las U-Mann-Whitney y KruskalWallis para las de distribución no normal. Las variables cualitativas se compararon mediante la prueba Chi-cuadrado. Con el propósito de identificar la relación entre ambos cuestionarios y el nivel de condición física, se creó una puntuación total de la batería (variable ZSFT), mediante la obtención de los valores individuales de los residuales ajustados de cada parámetro (transformando los valores de la prueba de agilidad y equilibrio dinámico (8-foot up- and-go en negativo) y sumando los seis componentes ${ }^{17}$.

La concordancia entre los valores de MMS estimados por el IPAQ y el VREM se va- loró mediante el método de Bland-Altman ${ }^{18}$, teniendo en cuenta que ninguna de las dos variables sigue una distribución normal ${ }^{19}$, para lo que se halló la diferencia entre sus valores individuales y se calculó la media y DE. Se calculó el intervalo de concordancia (rango de valores comprendidos entre la media $\pm 1,96 \mathrm{DE}$ ) y se representó gráficamente la relación entre la diferencia y la media de ambas variables con un diagrama de dispersión. Al observase que el cuestionario IPAQ-E no identificada a ningún sujeto en la categoría de AF baja, se utilizó el punto de corte de la Organización Mundial de la Salud (OMS) de 150 minutos/semana de AF moderada y/o vigorosa, para diferenciar entre participantes activos e inactivos ${ }^{20}$. Los análisis se realizaron con el programa SPSS v.15, considerando significativo un valor de $\mathrm{p}<0,05$.

\section{RESULTADOS}

De las 143 personas inscritas en el programa de mantenimiento, 105 participaron en esta investigación, 84,8\% mujeres con una edad media de 76,77 (DE=6,01) años. La muestra definitiva permitió detectar diferencias de $720 \mathrm{MET}$, con el nivel de significación y potencia predefinido. Las características sociodemográficas de los participantes, así como los valores obtenidos en la batería SFT, se muestran en la tabla 1. No se encontraron diferencias significativas en cuanto a la edad, el IMC y la condición física entre ambos sexos, a excepción de la flexibilidad, con valores significativamente mayores para las mujeres.

En la tabla 2 se muestran los valores obtenidos en ambos cuestionarios. El gasto total estimado por el VREM se situó en 5.382,48 (DE=2.611,75) MMS, no habiendo relación entre la edad y la cantidad de $\mathrm{AF}$ realizada. De acuerdo con los resultados de este cuestionario, el $88,5 \%$ de la muestra se consideró muy activa, el 10,3\% activa y el $1,2 \%$ sedentaria.

La AF total registrada para la muestra en general por el cuestionario IPAQ-E fue de 5.179,37 (1.457,58) MMS. Se observó que, con la edad, aumentó el tiempo sentado 
Tabla 1. Características generales y nivel de condición física de los participantes

\begin{tabular}{|c|c|c|c|}
\hline & Total & Hombres & Mujeres \\
\hline & 105 & $16(15,2 \%)$ & $89(84,8 \%)$ \\
\hline & Media (DE) & Media (DE) & Media (DE) \\
\hline \multicolumn{4}{|l|}{ Características } \\
\hline Edad (años) & $76,77(6,01)$ & $79,13(5,32)$ & $76,35(6,06)$ \\
\hline Grupos de edad (años) ${ }^{a}$ & $17(16,35)$ & $1(6,25)$ & $16(18,18)$ \\
\hline $65-69$ & $33(31,73)$ & $3(18,75)$ & $30(34,09)$ \\
\hline $70-74$ & $25(24,04)$ & $6(37,5)$ & $19(21,59)$ \\
\hline $75-79$ & $29(27,88)$ & $6(37,5)$ & $23(26,14)$ \\
\hline$>80$ & $154,28(7,3)$ & $162,9(8,03)$ & $152,69(5,96)$ \\
\hline Peso (kg) & $68,62(11,7)$ & $76,97(10,53)$ & $67,08(11,3)$ \\
\hline $\mathrm{IMC}\left(\mathrm{kg} / \mathrm{m}^{2}\right)$ & $28,78(4,19)$ & $29,01(3,62)$ & $28,75(4,31)$ \\
\hline \multicolumn{4}{|l|}{ Condición física } \\
\hline SFT I & $14,28(2,92)$ & $14,53(2,64)$ & $14,23(2,98)$ \\
\hline SFT II & $15,07(3,34)$ & $15,13(3,8)$ & $15,06(3,28)$ \\
\hline SFT III & $1,24(9,41)$ & $-4,73(8,27)$ & $2,32(9,23)^{*}$ \\
\hline SFT IV & $73,11(16,15)$ & $79,87(24,44)$ & $71,88(13,99)$ \\
\hline SFT V & $-10,21(14,71)$ & $-19,6(20,3)$ & $-8,51(12,91)^{*}$ \\
\hline SFT VI & $5,39(1,13)$ & $5,46(1,7)$ & $5,37(1,01)$ \\
\hline Total $^{* *}$ & $0,01(3,53)$ & $-0,82(5,06)$ & $0,16(3,2)$ \\
\hline
\end{tabular}

DE: desviación estándar; a: n (\%); IMC: índice de masa corporal; SFT: Senior Fitness Test; SFT I: fuerza de tren inferior; SFT II: fuerza de tren superior; SFT III: flexibilidad de tren inferior; SFT IV: capacidad cardiorrespiratoria; SFT V: flexibilidad de tren superior; SFT VI: agilidad/equilibrio dinámico; *: diferencia significativa entre sexos (t-Student); **: el total se calculó sumando los residuales ajustados de cada uno de los seis componentes una vez transformados los valores de la prueba SFT VI (agilidad y equilibrio dinámico) en negativo.

( $\mathrm{p}=0,003)$ y disminuyó el gasto energético $(p=0,011)$ (información no mostrada en tabla). De acuerdo con el punto de corte establecido por la OMS, el 52,4\% de la muestra se consideró activa.

El grado de concordancia encontrado entre ambos cuestionarios se muestra en la figura 1. Aunque a priori se observó una cierta correlación entre los mismos $(\mathrm{r}=0,447, \mathrm{p}<0,001)$, la diferencia media entre ambos fue no significativa (151,36 MMS, $\mathrm{DE}=2.370,67$, t-Student $\mathrm{p}=0,553)$. Debido a la gran amplitud encontrada en el rango de diferencias (-4495 a 4798) se consideró que los cuestionarios mostraron un grado de concordancia escaso. Atendiendo a la correlación entre la diferencia y la media $(\mathrm{r}=0,508, \mathrm{p}<0,001$, al aumentar los valores mayores son las diferencias positivas), se observó que el cálculo de MET fue sobreestimado para los valores altos por el VREM y para los bajos por el IPAQ-E. Por lo tanto, no se pudo crear una ecuación de regresión precisa entre ambos, ni pueden considerarse como herramientas de valoración intercambiables.

Se encontraron asociaciones significativas entre la condición física global y los ítems del VREM relativos al gasto energético derivado de caminar $(r=0,227)$ o 
Tabla 2. Puntuaciones obtenidas en los cuestionarios VREM e IPAQ-E

\begin{tabular}{|c|c|c|c|c|c|}
\hline & \multirow[b]{3}{*}{$\mathbf{n}$} & \multicolumn{4}{|c|}{ Puntuación } \\
\hline & & \multicolumn{2}{|c|}{ Tiempo (min/día) } & \multicolumn{2}{|c|}{ MMS } \\
\hline & & Mediana (RIC) & Media (DE) & Mediana (RIC) & Media (DE) \\
\hline \multicolumn{6}{|l|}{ VREM } \\
\hline Caminar & 86 & $60(60-120)$ & $87,15(66,92)$ & $1.450(1142-2900)$ & $1.987,19(1.518,64)$ \\
\hline Trabajar en el huerto & 20 & $180(60-240)$ & $178,5(122,92)$ & $1.105(673-2494)$ & $1.656,41(1.208,09)$ \\
\hline Deporte* & 87 & $45(45-83)$ & $84,99(64,38)$ & $570(380-810)$ & $733,54(590,96)$ \\
\hline Subir escaleras** & 48 & $6(3,25-10)$ & $8,29(8,32)$ & $88(44-215)$ & $157,11(185,17)$ \\
\hline Compras a pie & 77 & $180(120-240)$ & $197,14(161,63)$ & $810(540-1080)$ & $887,14(727,31)$ \\
\hline Limpiar la casa & 79 & $420(210-840)$ & $477,91(366,69)$ & $1.386(693-2772)$ & $1.577,11(1.210,06)$ \\
\hline Actividad Total & 87 & & & $5.030(3507-7004)$ & $5.382,48(2.611,75)$ \\
\hline \multicolumn{6}{|l|}{ IPAQ-E } \\
\hline Sedestación & & $360(240-450)$ & $355,33(140,25)$ & & \\
\hline Caminar $>10$ min & 105 & $420(285-525)$ & $396,73(160,96)$ & $4.158(4.158-4.158)$ & $4.008,24(539,24)$ \\
\hline AF moderada & 105 & $45(45-60)$ & $66,07(46,75)$ & $600(360-960)$ & $966,93(1.087,62)$ \\
\hline AF vigorosa & 14 & $120(30-210)$ & $133,57(112,43)$ & $1.440(480-2.160)$ & $1.531,43(1.104)$ \\
\hline AF moderada/vigorosa & 105 & $160(90-245)$ & $282,45(354,76)$ & & \\
\hline Actividad total & 105 & & & $4.758(4.518-5.118)$ & $5.179,37(1.457,58)$ \\
\hline Activo*** $^{* *}$ & 105 & $55(52,4 \%)$ & & & \\
\hline
\end{tabular}

MMS: MET-minuto-semana; min/día: minutos por día; RIC: rango intercuartil; DE: desviación estándar; VREM: versión reducida en español del cuestionario de actividad física en el tiempo libre de Minnesota; IPAQ-E: cuestionario internacional de actividad física adpatado a personas mayores; MET: tasa metabólica equivalente; RIC: rango intercuartil; *: número de practicantes por deporte: baile: 13 , bicicleta: 2 , cachibol: 1 , ejercicio en casa: 4 , gimnasia: 87 , natación: 22 , yoga: $3 ;{ }^{* *}$ : pisos/día; AF: actividad física; ${ }^{* * *}$ : clasificación de actividad según la OMS.

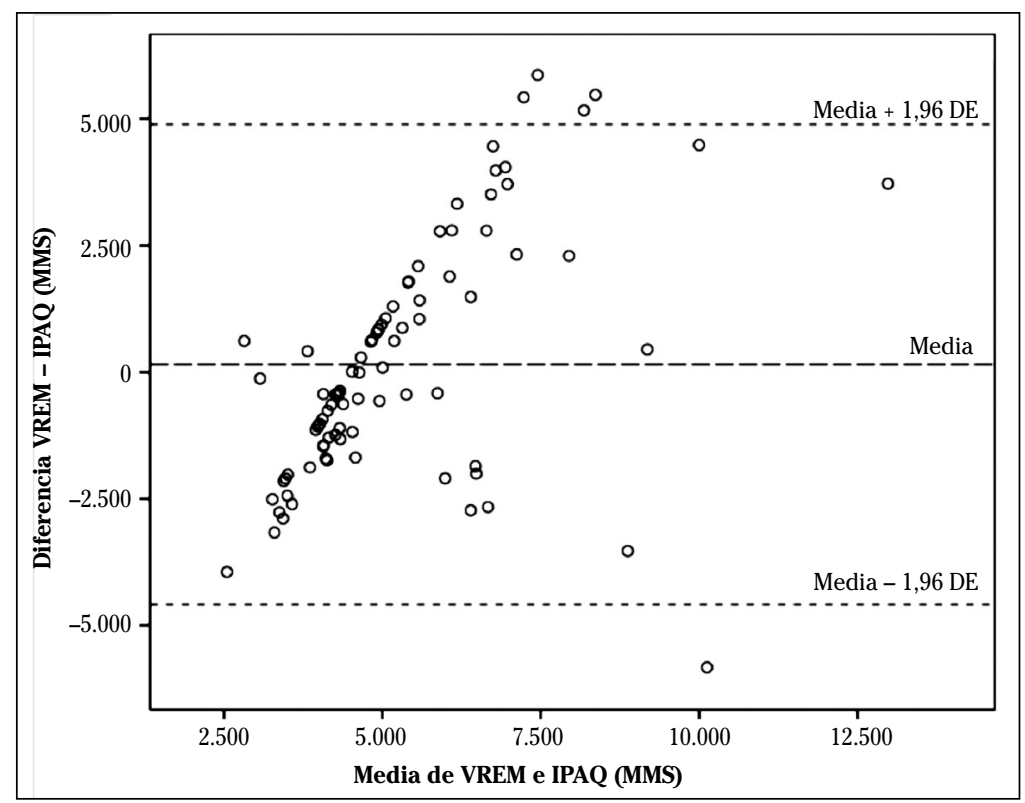

Figura 1. Gráfico de dispersión de la concordancia del cálculo de MMS entre el VREM y el IPAQ-E, según el método de Bland-Altman. 


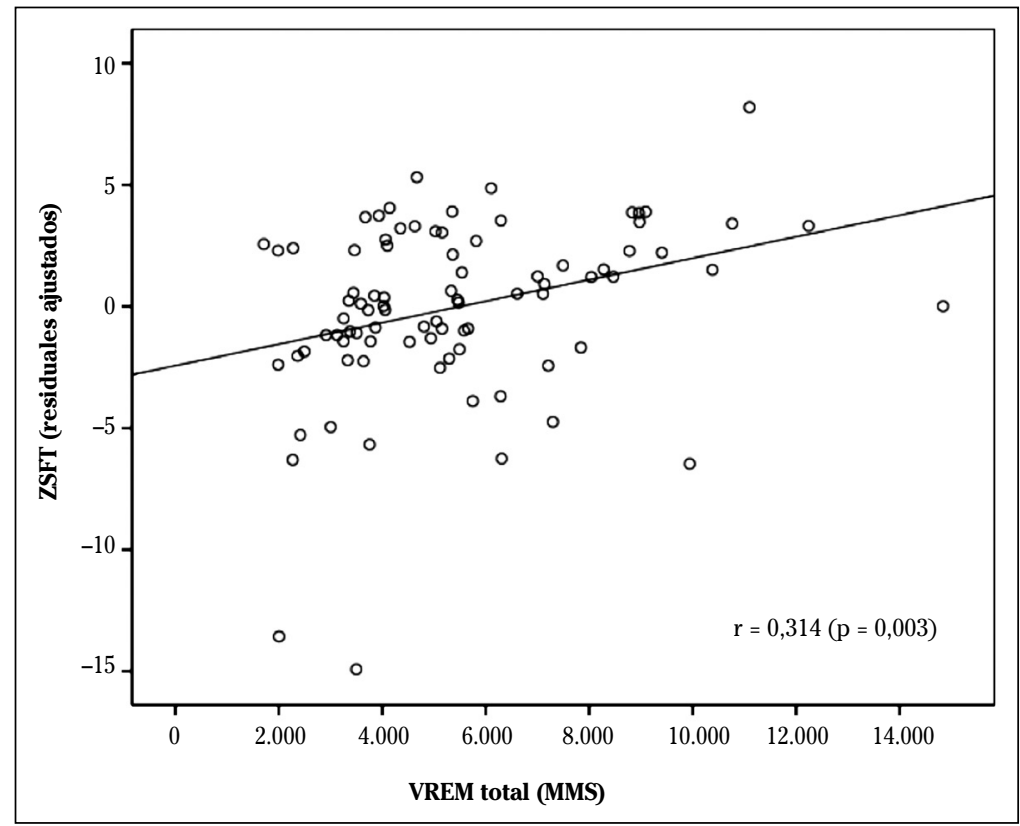

* La variable ZSFT se calculó sumando los residuales ajustados de cada uno de los seis
componentes, una vez transformados los valores de la prueba SFT VI (agilidad y equilibrio
dinámico) en negativo. MMS: MET-minuto-semana.

Figura 2. Gráfico de dispersión de la relación entre los MET-minuto-semana estimados con el VREM y la puntuación total* de la batería Senior Fitness Test.

de realizar actividades deportivas o baile $(\mathrm{r}=0,235)$, y el gasto energético total estimado ( $r=0,314$ ) (Fig. 2). No se observó ningún tipo de asociación significativa entre el IPAQ-E y la condición física, ni a nivel global ni para cada uno de los componentes individuales de ambos.

$\mathrm{Al}$ comparar los niveles de $\mathrm{AF}$ por las categorías obtenidas con el VREM, se encontró que los sujetos muy activos presentaron mejor condición física que los activos o sedentarios. La condición física global de los sujetos considerados activos en base a la cantidad de AF semanal estimada por el IPAQ-E y tras aplicar el punto de corte de la OMS, fue significativamente superior a la de los inactivos (Tabla 3).

\section{DISCUSIÓN}

En este estudio de la relación entre dos cuestionarios diseñados para registrar la cantidad de AF estimada y el nivel de $\mathrm{CF}$ en una muestra de personas mayores de 65 años se incluyó además el IMC, componente de la $\mathrm{CF}$ que ha sido empleado como variable de comparación en estudios similares ${ }^{7}$.

El VREM se creó a partir del Cuestionario de actividad física en el tiempo libre de Minnesota, una herramienta de estimación de la AF diseñada para población adulta, que fue el único instrumento utilizado en el estudio desarrollado para determinar su validez. Por lo tanto, los resultados de la presente investigación contribuyen a incrementar la evidencia científica al respecto de las propiedades psicométricas de este cuestionario. Tras el análisis estadístico realizado, se observaron correlaciones significativas entre varios ítems del VREM y su puntuación total con distintos componentes de la CF de los participantes, así como con el sumatorio de la batería SFT. Si bien dichas correlaciones fueron débiles, 
Tabla 3. Relación entre la condición física estimada por el SFT y el nivel de actividad estimada por los cuestionarios VREM e IPAQ-E

\begin{tabular}{|c|c|c|c|c|}
\hline \multicolumn{3}{|c|}{ VREM } & & \multirow[b]{2}{*}{$\begin{array}{c}\text { Diferencia de medias } \\
\text { (IC 95\%) }\end{array}$} \\
\hline Condición física & $\begin{array}{l}\text { Muy Activo } \\
\quad(\mathrm{n}=77) \\
\text { Media }(D E)\end{array}$ & $\begin{array}{c}\text { Activo o sedentario } \\
(\mathrm{n}=10) \\
\text { Media }(D E)\end{array}$ & p & \\
\hline SFT I & $14,57(2,41)$ & $11,30(4,42)$ & 0,045 & $3,27(0,08 ; 6,46)$ \\
\hline SFT II & $15,43(3,13)$ & $13,00(4,37)$ & 0,031 & $2,43(0,23 ; 4,63)$ \\
\hline SFT III & $0,55(9,51)$ & $2,75(9,71)$ & 0,494 & $-2,20(-8,57 ; 4,17)$ \\
\hline SFT IV & $74,48(15,61)$ & $61,89(22,05)$ & 0,031 & $12,59(1,15 ; 24,03)$ \\
\hline SFT V & $-10,15(14,55)$ & $-11,20(18,14)$ & 0,835 & $1,05(-8,95 ; 11,06)$ \\
\hline SFT V: & $5,32(0,98)$ & $5,79(2,03)$ & 0,492 & $-0,47(-1,93 ; 1)$ \\
\hline Total $^{*}$ & $0,28(3,29)$ & $-2,69(5,22)$ & 0,019 & $2,97(0,5 ; 5,43)$ \\
\hline \multicolumn{3}{|c|}{ IPAQ-E } & & \multirow[b]{2}{*}{$\begin{array}{c}\text { Diferencia de medias } \\
\text { (IC } 95 \%)\end{array}$} \\
\hline Condición física & $\begin{array}{c}\text { Activo } \\
(\mathrm{n}=52) \\
\text { Media }(D E)\end{array}$ & $\begin{array}{c}\text { Inactivo } \\
(\mathrm{n}=46) \\
\text { Media }(D E)\end{array}$ & $\mathbf{p}$ & \\
\hline SFT II & $15,38(2,76)$ & $14,72(3,9)$ & 0,327 & $-0,67(-2,01 ; 0,68)$ \\
\hline SFT III & $2,89(8,35)$ & $-0,62(10,24)$ & 0,065 & $-3,51(-7,24 ; 0,22)$ \\
\hline SFT IV & $75,65(14,87)$ & $70,18(17,21)$ & 0,096 & $-5,48(-11,94 ; 0,99)$ \\
\hline SFT V & $-8,07(13,69)$ & $-12,62(15,59)$ & 0,127 & $-4,55(-10,42 ; 1,32)$ \\
\hline SFT VI & $5,20(0,68)$ & $5,60(1,47)$ & 0,094 & $0,40(-0,07 ; 0,87)$ \\
\hline Total* & $0,79(2,58)$ & $-0,89(4,23)$ & 0,023 & $-1,69(-3,14 ;-0,24)$ \\
\hline
\end{tabular}

VREM: versión reducida en español del cuestionario de actividad física en el tiempo libre de Minnesota; DE: deviación estándar; IC 95\%: intervalo de confianza al 95\% para la diferencia de medias; IPAQ-E: cuestionario internacional de actividad física adaptado a personas mayores; SFT: senior fitness test; SFT I: fuerza de tren inferior; SFT II: fuerza de tren superior; SFT III: flexibilidad de tren inferior; SFT IV: capacidad cardiorrespiratoria; SFT V: flexibilidad de tren superior; SFT VI: agilidad/equilibrio dinámico; *: el total se calculó sumando los residuales ajustados de cada uno de los seis componentes una vez transformados los valores de la prueba SFT VI (agilidad y equilibrio dinámico) en negativo.

la existencia de este tipo de asociaciones está en línea con lo reportado para otros cuestionarios. Así, las versiones españolas del cuestionario de Yale, de Baecke, y del cuestionario de valoración de la $\mathrm{AF}$ en el tiempo libre (LTPAI) correlacionaron significativamente con los resultados de la prueba de los seis minutos, un indicador de la capacidad cardiorrespiratoria de las personas mayores ${ }^{7}$. En esta línea, otros cuestionarios diseñados para estimar la cantidad de AF realizada por la tercera edad, como la versión brasileña del cuestionario de Godin-Shephard ${ }^{12}$, o la versión original del cuestionario CHAMPS $^{21}$ mostraron asociaciones significativas con dimensiones de la
CF como la capacidad cardiorrespiratoria, la fuerza o la agilidad.

Con relación al IPAQ-E, en general no se encontraron asociaciones significativas entre ninguna de las variables analizadas, lo que es un hallazgo inesperado. Así, la versión inglesa de este cuestionario, sí mostró correlaciones significativas con distintos componentes de la $\mathrm{CF}$, como la resistencia aeróbica o la fuerza ${ }^{22}$, mientras que la versión española se asoció significativamente con el equilibrio y la movilidad ${ }^{10}$. Los datos analizados en la presente investigación indican que el IPAQ-E no sigue esta tendencia y por lo tanto su validez convergente no se considera adecuada. Existen otros estudios 
en los que la validez de este cuestionario también ha sido puesta en duda. Así, la versión brasileña mostró una ausencia de asociación significativa con los datos registrados a través de podómetro ${ }^{23}$ mientras que, recientemente, Ryan y coll $^{24}$, tras comparar las puntuaciones obtenidas en la versión inglesa con los datos registrados por acelerómetros, concluyeron que el IPAQ no es una herramienta válida para estimar la cantidad de $\mathrm{AF}$ realizada por las personas mayores.

Otro hecho destacable del IPAQ-E es la ausencia de correlación entre el ítem de valoración tiempo sentado y la CF. El tiempo sentado es una medida de gran importancia, especialmente en la tercera edad, al ser un indicador del nivel de sedentarismo, pero de difícil estimación en esta población ${ }^{25}$. Sin embargo, otras versiones de este cuestionario si mostraron asociaciones significativas entre el tiempo sentado y la cantidad de AF valorada a través de acelerómetros en personas mayores de 65 $\operatorname{años}^{8,26,27}$, mientras que en el estudio de validación al castellano no se informó sobre las asociaciones establecidas por este ítem ${ }^{10}$. Los resultados de la presente investigación aconsejan prudencia a la hora de estimar mediante el empleo de este cuestionario la cantidad de tiempo que las personas mayores pasan sentadas.

De acuerdo a lo hasta aquí recogido, el IPAQ-E parece presentar cierta incertidumbre en lo que a su validez se refiere, tal y como se ha observado para la versión originalmente creada para población adulta ${ }^{28}$. De todos modos, es interesante resaltar que el cuestionario mostró una mayor sensibilidad cuando la muestra se estratificó según su nivel de AF de acuerdo con los puntos de corte establecidos por la OMS. Por lo tanto, y en consonancia con otros estudios ${ }^{8}$, el IPAQ-E podría ser útil para clasificar a las personas mayores en distintas categorías de AF, dependiendo de la cantidad de tiempo en minutos que estiman realizarla.

Tanto el VREM como el IPAQ-E indicaron que gran parte de la muestra era muy activa, si bien -a juzgar por los resultados logrados en la SFT- los participantes en este estudio presentaron una CF más baja de lo que se podría esperar ${ }^{29}$. Esta ausen- cia de concordancia por parte de ambos cuestionarios podría deberse a una posible tendencia por parte de los encuestados a sobrestimar la cantidad de AF vigorosa y subestimar la duración e intensidad de las actividades sedentarias, sesgo que se ha sugerido que presentan este tipo de herramientas ${ }^{7}$. A pesar de ello, el VREM sí mostró ciertas correlaciones significativas con algunos componentes de la $\mathrm{CF}$, al contrario de lo que ocurrió con el IPAQ-E. La mayor precisión del VREM podría deberse a su formato, pues se ha observado que la sensibilidad del IPAQ-E se ve influenciada por los problemas que presentan las personas mayores para responder a preguntas de carácter abierto, siendo recomendable presentar preguntas de carácter más cerrado y con ejemplos concretos de activida$\operatorname{des}^{26}$, aspectos que cumple en mayor medida el VREM. Esto es especialmente cierto para el ítem relacionado con el tiempo que se emplea en caminar, de carácter mucho más abierto en el IPAQ, mientras que en el VREM la cuestión es enunciada de un modo más específico. Esta disparidad a la hora de registrar una misma actividad puede ser la causa de la gran diferencia observada en el resultado de este ítem cuando se comparan ambos cuestionarios.

En general, los datos de este estudio apuntan a que el empleo del VREM es preferible al del IPAQ-E, si bien existen ciertas debilidades metodológicas inherentes a las características de la muestra incluida en el mismo, que deben ser reconocidas. Así, el número de participantes no fue lo suficientemente grande como para poder realizar un análisis estratificado por edad. Además, el porcentaje de hombres fue muy reducido, lo que limita la comparación entre sexos. Finalmente, el hecho de que la muestra estuviese conformada por usuarios de un programa de gimnasio de mantenimiento que no presentaban problemas de salud relevantes, limita la extrapolación de los resultados aquí presentados a la población geriátrica en general.

En conclusión, cuando se pretenda estimar la cantidad de AF realizada por las personas mayores mediante los cuestionarios VREM o IPAQ-E, se aconseja el empleo 
del primero, por presentar cierto grado de asociación, si bien débil, con el nivel de CF de esta población. Son necesarios futuros estudios que incluyan herramientas de valoración objetiva del gasto energético, para determinar el grado de validez que ambos cuestionarios presentan.

\section{BIBLIOGRAFÍA}

1. Onerup A, Arvidsson D, Blomevist $\AA$, Daxberg EL, JivegåRD L, JonsDotTiR IH et al. Physical activity on prescription in accordance with the Swedish model increases physical activity: a systematic review. Br J Sports Med 2019; 53: 383-388. https://doi.org/10.1136/ bjsports-2018-099598

2. Fishman EI, Steeves JA, Zipunnikov V, Koster A, BeRRIGAN D, HARRIS TA et al. Association between objectively measured physical activity and mortality in NHANES. Med Sci Sports Exerc 2016; 48: 1303-1311. https://doi.org/10.1249/ MSS. 0000000000000885

3. Hamer M, de Oliveira C, Demakakos P. Non-exercise physical activity and survival: English longitudinal study of ageing. Am J Prev Med 2014; 47: 452-460. https://doi.org/10.1016/j. amepre.2014.05.044

4. Casas-Herrero A, Izquerdo M. Physical exercise as an efficient intervention in frail elderly persons. An Sist Sanit Navar 2012; 35: 69-85. https://doi.org/10.4321/s113766272012000100007

5. Skender S, Ose J, Chang-Claude J, Paskow M, Brühmann B, SIEgel EM et al. Accelerometry and physical activity questionnaires-a systematic review. BMC public health 2016; 16(1): 515. https://doi.org/10.1186/s12889-016-3172-0

6. Martínez-Lemos RI, Ayán Pérez C, Sánchez Lastra A, Cancela Carral JM, Valcarce Sánchez R. Cuestionarios de actividad física para niños y adolescentes españoles: una revisión sistemática. An Sist Sanit Navar 2016; 39: 417-428. https://doi.org/10.23938/ASSN.0232

7. Sánchez-Lastra MA, Martínez-Lemos I, Cancela JM, Aragón Cancela C, Ayán C. Cuestionarios de estimación de actividad física: revisión sistemática y análisis de sus propiedades psicométricas en población española mayor de 60 años. Rev Esp Salud Pública 2018; 92: e1-e17. https://doi.org/10.1590/s113557272011000500009

8. Hurtig-Wennlof A, Hagstromer M, Olsson la. The International Physical Activity Ques- tionnaire modified for the elderly: aspects of validity and feasibility. Public Health Nutr 2010; 13: 1847-1854. https://doi.org/10.1017/ s1368980010000157

9. Ruiz Comellas A, Pera G, Baena Díez JM, Mundet Tudurí X, Alzamora Sas T, Elosua R et al. Validación de una versión reducida en español del cuestionario de actividad física en el tiempo libre de Minnesota (VREM). Rev Esp Salud Pública 2012; 86: 495-508. https://doi. org/10.4321/S1135-57272012000500004

10. Rubio Castañeda FJ, Tomás Aznar C, Muro BaQUERo C. Medición de la actividad física en personas mayores de 65 años mediante el IPAQ-E: validez de contenido, fiabilidad y factores asociados. Rev Esp Salud Pública 2017; 91. https://doi.org/10.4321/s113557272015000600003

11. Fogelhom M, Malmberg J, Suni J, Santtila M, KYRÖLÄINEN H, MänTYSAARI M et al. International Physical Activity Questionnaire: validity against fitness. Med Sci Sports Exerc 2006; 38: 753-760. https://doi.org/10.1249/01. mss.0000194075.16960.20

12. SÃo-João TM, Rodrigues RC, Gallani MM, Miura CT, Domingues Gde B, Godin G. Cultural adaptation of the Brazilian version of the Godin-Shephard Leisure-Time Physical Activity Questionnaire. Rev Saude Publica 2013; 47: 479-487. https://doi.org/10.1590/s00348910.2013047003947

13. Cancela JM, Ayán C, Sánchez-Lastra Ma, Casal A. Construct validity of the spanish version of the CHAMPS questionnaire for institutionalized patients over 65 years old. Motriz 2017; 23: 81-87. https://doi.org/10.1590/s19806574201700010013

14. Hills AP, StREet SJ, Byrne NM. Physical activity and health: "what is old is new again". Adv Food Nutr Res 2015; 75: 75-95.

15. Rikцi R, Jones C. Senior fitness test manual. Estados Unidos de América. Human Kinetics 2001.

16. CHAN YH. Biostatistics 104: correlational analysis. Singapore Med J 2003; 44: 614-619.

17. Cancela JM, Ayán C, Gutiérrez-Santiago A, Prieto I, Varela, S. The Senior Fitness Test as a functional measure in Parkinson's disease: a pilot study. Parkinsonism Relat Disord 2012; 18: 170-173. https://doi.org/10.1016/j.parkreldis.2011.09.016

18. BLAND JM, ALtman DG. Statistical methods for assessing agreement between two methods of clinical measurement. Lancet 1986; 1: 307-310. https://doi.org/10.1016/s0140-6736(86)90837-8

19. Bland JM, Altman DG. Measuring agreement in method comparison studies. Stat 
Methods Med Res 1999; 8: 135-160. https:// doi.org/10.1191/096228099673819272

20. Global recommendations on physical activity for health. World Health Organization 2010; Recuperado de: https://www.who.int/ dietphysicalactivity/factsheet_olderadults/ en/

21. Cyarto EV, Marshall AL, Dickinson RK, Brown WJ. Measurement properties of the CHAMPS physical activity questionnaire in a sample of older Australians. J Sci Med Sport 2006; 9: 319-326. https://doi.org/10.1016/j. jsams.2006.03.001

22. Baldwin JN, McKay MJ, Hiller CE, Moloney N, Nightingale EJ, Burns J. Relationship between physical performance and self-reported function in healthy individuals across the lifespan. Musculoskelet Sci Pract 2017; 30: 10-17. https://doi.org/10.1016/j.msksp.2017.05.001

23. Benedetti TRB, Antunes PDC, Rodriguez-AÑez CR, Mazo GZ, Petroski EL. Reprodutibilidade e validade do Questionário Internacional de Atividade Física (IPAQ) em homens idosos. Rev Bras Med Esporte 2007; 13: 11-16. https:// doi.org/10.1590/s1517-86922007000100004

24. Ryan DJ, Wullems JA, Stebbings GK, Morse CI, Stewart CE, Onambele-Pearson GL. Reliability and validity of the international physical activity questionnaire compared to calibrated accelerometer cut-off points in the quantification of sedentary behaviour and physical activity in older adults. PLoS One 2018; 13 : e0195712. https://doi.org/10.1371/journal. pone.0195712

25. Van Uffelen JG, Heesch KC, Hill RL, Brown WJ. A qualitative study of older adults' responses to sitting-time questions: do we get the information we want? BMC Public Health 2011; 11: 458. https://doi.org/10.1186/1471-2458$11-458$

26. Tоmioka K, Iwamoto J, Saeki K, Okamoto N. Reliability and validity of the International Physical Activity Questionnaire (IPAQ) in elderly adults: the Fujiwara-kyo Study. J Epidemiol 2011; 21: 459-465. https://doi.org/10.2188/jea. je20110003

27. Wanner M, Probst-Hensch N, Kriemler S, Meier F, Autenrieth C, Martin BW. Validation of the long international physical activity questionnaire: influence of age and language region. Prev Med Rep 2016; 3: 250-256. https://doi. org/10.1016/j.pmedr.2016.03.003

28. Lee PH, Macfarlane DJ, Lam T, Stewart SM. Validity of the international physical activity questionnaire short form (IPAQ-SF): a systematic review. Int J Behav Nutr Phys Act 2011; 8:115. https://doi.org/10.1186/1479-5868-8115

29. Pedrero-Chamizo R, Gomez-Cabello A, Delgado S, Rodríguez-Llarena S, Rodríguez-Marroyo JA, CABANILLAS E et al. Physical fitness levels among independent non-institutionalized Spanish elderly: the elderly EXERNET multi-center study. Arch Gerontol Geriatr 2012; 55: 406-416. https://doi.org/10.1016/j.archger.2012.02.004 
